\title{
Measurement of Dynamic Retention with Fast Ejecting System of Targeted Sample (FESTA)*)
}

\author{
Qilin YUE, Kazuaki HANADA ${ }^{1)}$, Makoto OYA ${ }^{2)}$, Shogo MATSUO, Shinichiro KOJIMA, \\ Hiroshi IDEI $^{1)}$, Takumi ONCHI ${ }^{1)}$, Kengoh KURODA ${ }^{1)}$, Naoaki YOSHIDA ${ }^{1)}$, Ryuya IKEZOE ${ }^{1)}$, \\ Yukai LIU ${ }^{3)}$, Makoto HASEGAWA ${ }^{1)}$, Shun SHIMABUKURO ${ }^{1)}$, Aki HIGASHIJIMA ${ }^{1)}$, \\ Takahiro NAGATA ${ }^{1)}$ and Shoji KAWASAKI ${ }^{1)}$ \\ Interdisciplinary Graduate School of Engineering Sciences, Kyushu University, Kasuga 816-8580, Japan \\ ${ }^{1)}$ Research Institute for Applied Mechanics, Kyushu University, Kasuga 816-8580, Japan \\ ${ }^{2)}$ Faculty of Engineering Sciences, Kyushu University, Kasuga 816-8580, Japan \\ ${ }^{3)}$ Institute of Plasma Physics, Chinese Academy of Sciences, Hefei 230031, China
}

(Received 27 November 2019 / Accepted 24 February 2020)

\begin{abstract}
Fast Ejecting System of Targeted sAmple called FESTA has been developed to carry out the measurement of dynamic hydrogen retention by a test sample. A sample can be exposed and extracted from the targeted plasma at any time using FESTA, however, when exposing the sample, the test chamber wall gets coated by some hydrogen as it is open to the QUEST vacuum vessel. We refer to this as the plasma-induced background. To measure the amount of hydrogen retained by the sample itself, the contribution from the plasma induced background must be subtracted from the measurements. To measure the accurate dynamic retention from plasma-exposed sample, a background subtraction model has been developed and tested. The initial testing shows that the FESTA system and model can estimate the dynamic hydrogen retention by a target test sample.
\end{abstract}

(C) 2020 The Japan Society of Plasma Science and Nuclear Fusion Research

Keywords: dynamic retention, hydrogen recycling, plasma wall interaction, plasma-induced background, FESTA

DOI: $10.1585 /$ pfr.15.2402013

\section{Introduction}

It is important for tokamaks-based experiments to demonstrate steady state operation (SSO) to realize economical nuclear fusion power generation. To reduce the amount of wall-stored tritium which is used as a fuel in nuclear fusion reaction and because tritium is radioactive, metals such as tungsten are now being adopted for plasma facing wall (PFW) components, instead of carbon which has been used in many earlier tokamak experiments [1,2]. Recently, it was reported that there was a dramatic reduction in wall-stored fuel particles during ITERlike wall (ILW) experiments using such metallic PFWs in JET [3-6]. Such a reduction of wall-stored fuel particles clearly indicates that dynamic retention of fuel particles by the vessel walls greatly enhances fuel recycling and plays a significant role in fuel particle balance.

QUEST (Q-shu University Experiment with Steady Spherical Tokamak) is equipped with all metallic $[1,7,8]$ and temperature-controlled PFWs, and has the capability to achieve steady-state operation during long-discharges lasting more than one hour [1]. In QUEST experiments, it is found that a redeposited layer of several tens $\mathrm{nm}$ in thickness due to plasma wall interaction (PWI) also plays

author'se-mail: k.gaku@triam.kyushu-u.ac.jp

*) This article is based on the presentation at the 28th International Toki Conference on Plasma and Fusion Research (ITC28). a critical role in fuel particle balance $[9,10]$.

So far, static retention has been quantitatively measured in QUEST [11], and dynamic retention was indirectly measured by nuclear reaction analysis (NRA) for plasma-exposed specimens [9, 12-15]. In the NRA method, the exposed sample is shipped to an external facility and bombarded by an ion beam for analysis [15]. This may not adequately reflect what happens in a tokamak experiment in which the walls are impacted by energetic hydrogen atoms. The data on dynamic retention by a wall facing a high temperature plasma is obtained as a global value, and it is difficult to understand the retention properties of the wall in relation to the surface conditions such as the thickness of redeposited layer. For a more realistic understanding of the properties of the redeposited layer, it would be better to conduct an in-situ analysis of a targeted sample exposed to the tokamak plasma.

In support of such an analysis, the FESTA system (Fast Ejecting System of Targeted sAmple) has been developed. In this system, a sample exposed to the QUEST plasma is withdrawn into a mechanically isolated chamber and its hydrogen retention properties measured immediately after the conclusion of a plasma experiment.

In this research, the programmable operation of the FESTA system using a LabVIEW software interface is tested to assess if the FESTA system can support plasma 


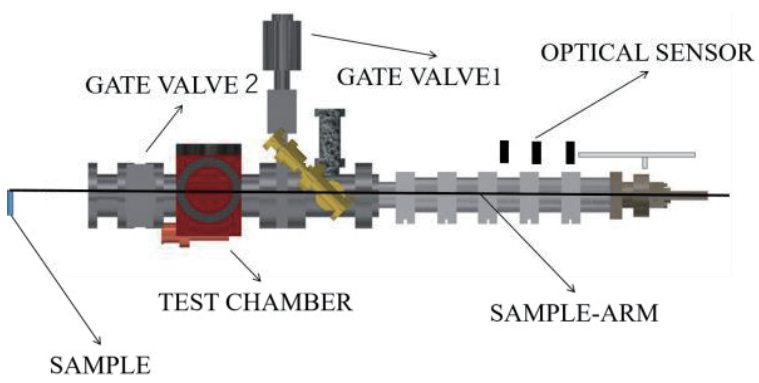

Fig. 1 Schematic of FESTA.

experiments, including in-situ sample analysis. One of the first observations was that the test chamber wall of FESTA receives a significant impact from plasma-induced $\mathrm{H}$ atoms which have higher energy than molecular $\mathrm{H}_{2}$ gas. A model for such plasma-induced particles was constructed to estimate the unexpected number of $\mathrm{H}_{2}$ particles that were emitted from the test chamber of FESTA. Finally, the dynamic retention from a plasma-exposed sample made of type SUS316L was quantitatively measured with FESTA.

\section{Operation of the FESTA System}

As shown in Fig. 1, FESTA is composed of two gate valves, a test chamber located between the gate valves, and a telescopic device which is connected to a movable sample-arm. The movable sample-arm is designed to be able to pick a sample and to expose it to the plasmas, and then to extract it quickly at the targeted time. The gate valves open and close based on signals from the optical sensors which monitor the sample-arm position. After the sample-arm has picked a plasma exposed sample and moved it to the test chamber, the gate valve 2 is closed. The plasma-exposed sample is left in the test chamber and then the sample-arm passes through gate valve 1 . The test chamber is isolated by closing gate valve 1 . Then, the partial pressure of $\mathrm{H}_{2}$ in the test chamber is monitored with a quadrupole mass spectrometer (QMS), which provides the dynamic $\mathrm{H}_{2}$ retention data of the test sample. All the needed steps are pre-programmed, and a sample analysis request can be executed as required for an experiment.

\section{Experimental Results}

\subsection{Evaluation of hydrogen retention in QUEST}

The macroscopic particle balance is given by the equation

$$
\frac{\mathrm{d} H_{\text {wall }}}{\mathrm{d} t}=\Gamma_{\text {in }}-\Gamma_{\text {out }}=\Gamma_{\text {wall }},
$$

where $H_{\text {wall }}$ denotes the number of the wall-stored hydrogen, $\Gamma_{\mathrm{in}}$ is the injected particle flux to the entire wall of QUEST, and $\Gamma_{\text {out }}$ is the emitted particle flux. $\Gamma_{\text {wall }}$ is the amount of particle flux occluded in the QUEST wall.

When the plasma discharge is terminated, the wall in-

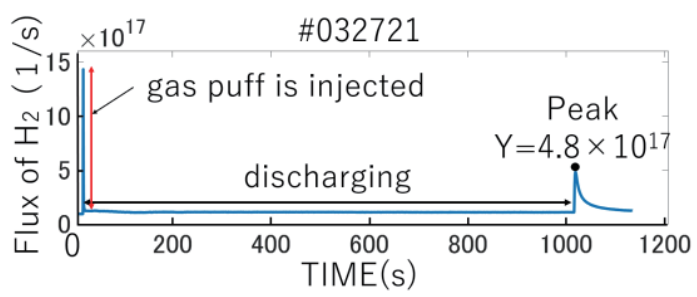

Fig. 2 Time evolution of hydrogen flux released from PFWs into the vacuum vessel.

jected particles disappears, so $\Gamma_{\text {in }}$ becomes zero. Finally, recombined hydrogen molecules are released from the wall into the vacuum vessel. A part of them $\left(H_{\mathrm{vv}}\right)$ stays in the vacuum vessel and the others $\left(H_{\text {pump }}\right)$ are exhausted by the vacuum pumps. Therefore, $\Gamma_{\text {out }}$ can be expressed by

$$
\Gamma_{\text {out }}=\frac{\mathrm{d} H_{\mathrm{vv}}+\mathrm{d} H_{\text {pump }}}{\mathrm{d} t} .
$$

When hydrogen flux released by the PFWs reaches its peak value, the value of $\mathrm{d} H_{\mathrm{vv}} / \mathrm{d} t$ is zero, so $\Gamma_{\text {out }}$ equals $\mathrm{d} H_{\text {pump }} / \mathrm{d} t$. As shown in Fig. 2, the ion current measured by the QMS is converted into exhausted flux using a calibrated flow meter and such a calibration is carried out at the start and end of every experimental day $[1,9]$. The hydrogen flux released from the wall of QUEST is about $\Gamma_{\text {out }}$ $4.8 \times 10^{17} \mathrm{~s}^{-1}$.

The amount of hydrogen particles occluded in the QUEST wall at the end of the plasma discharge is determined by integrating the experimental data from Eq. (1), and it is found to be about $N_{\text {retQ }} 2.3 \times 10^{19}$. The suffix 'Q', ' $\mathrm{C}$ ' and ' $\mathrm{S}$ ' in this paper indicate 'the QUEST vacuum vessel', 'the test chamber' and 'sample', respectively.

\subsection{Verification of measurements from FESTA}

Since the test chamber and the QUEST vacuum vessel are connected during plasma-exposure of the sample, particles such as $\mathrm{H}_{2}$ and other gases are freely passing through the gate valve 2 . When the gate valve 1 and 2 are closed, the $\mathrm{H}_{2}$ gas in the test chamber of FESTA leads to background $\mathrm{H}_{2}$ pressure $\left(N_{\text {backgC }}\right)$ which is the sum of the partial pressure from this pre-existing gas and the $\mathrm{H}_{2}$ partial pressure resulting from the $\mathrm{H}_{2}$ released by the sample. The background gas in the QUEST vessel ( $\left.N_{\text {backgQ }}\right)$ is about $2.6 \times 10^{16}$, under the assumption that the gas is assumed to be an ideal gas, their pressures are uniform, and the gas temperature can be regarded as the wall temperature, so $N_{\text {backgC }}\left(\mathrm{H}_{2}\right)$ is

$$
N_{\text {backgC }}=N_{\text {backQ }} \frac{V_{\mathrm{C}}}{V_{\mathrm{Q}}} \approx 9.3 \times 10^{12},
$$

where $V_{\mathrm{C}}=5 \times 10^{-3} \mathrm{~m}^{3}$ and $V_{\mathrm{Q}}=14 \mathrm{~m}^{3}$ are the volume of the test chamber of FESTA and the QUEST vacuum vessel respectively. Here, the suffix ' $Q$ ', ' $C$ ' and ' $S$ ' indicate 'the QUEST vacuum vessel', 'the test chamber' and 'sample', 


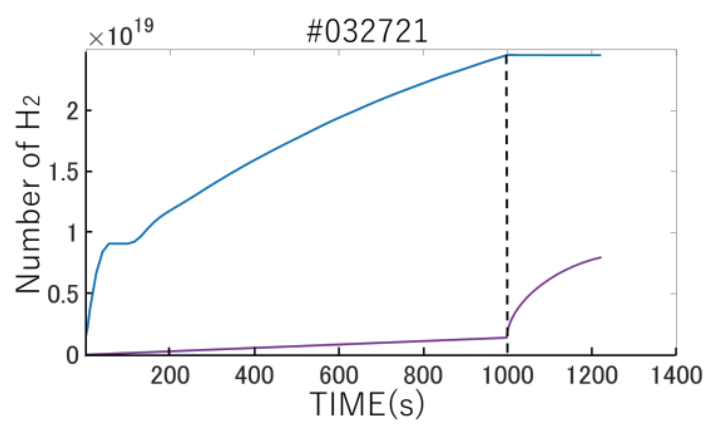

Fig. 3 Plot of the number of hydrogen particles injected into the QUEST vacuum vessel by mass flow (blue trace), and the amount pumped out (purple trace). The plasma discharge duration is $1000 \mathrm{~s}$.

respectively. In the FESTA experiment, the background $\mathrm{H}_{2}$ gas content in the test chamber is about $1.5 \times 10^{13}$, which is nearly the value calculated in Eq. 3. Based on the data shown in Fig. 3, the amount of hydrogen particles released from the QUEST vacuum vessel wall $N_{\text {retQ }}$ is the difference between the two traces so the amount $\left(\mathrm{H}_{2}\right)$ released from the sample is about:

$$
N_{\text {retS }}=N_{\text {retQ }} \frac{S_{\mathrm{S}}}{S_{\mathrm{Q}}} \approx 2.2 \times 10^{15} .
$$

Where $S_{\mathrm{S}}=2.5 \times 10^{-3} \mathrm{~m}^{2}$ and $S_{\mathrm{Q}}=26.6 \mathrm{~m}^{2}$ are the area of plasma-exposed sample and plasma-facing surface of the QUEST vacuum vessel. It could be concluded that the amount of hydrogen particles released from the sample $N_{\text {rets }}$ is hundred times more than the background $\mathrm{H}_{2}$ content in the test chamber $N_{\text {backgC }}$, so $N_{\text {backgC }}$ should be negligible.

Moreover, there also exists an outgassing flux $\Gamma_{\text {outC }}$ from the wall of test chamber. Generally, the amount of gas per unit time released from a vacuum vessel is related to

$$
\Gamma_{\text {outC }}-p S_{\text {pump }}=V \frac{d_{\mathrm{p}}}{d_{\mathrm{t}}},
$$

where $p$ is pressure of vessel, $S_{\text {pump }}$ is pumping speed of vacuum pump and $V$ is volume of vacuum vessel. As the test chamber is isolated during hydrogen pressure measurement, $S_{\text {pump }}$ becomes zero. On the other hand, $q$, the amount of released gas per unit time per area depends on the surface conditions and the material, so we estimate using $q$ for the conventional stainless type SUS304L, $q=$ $2.3 \times 10^{-8} \mathrm{~Pa} \cdot \mathrm{m}^{3} \cdot \mathrm{s}^{-1} \cdot \mathrm{m}^{-2}$ as given in [16]

$$
\Gamma_{\text {outc }}=q S_{\mathrm{C}} \approx 6.7 \times 10^{11}\left(\mathrm{~S}^{-1}\right) .
$$

Here, the surface area of the wall of test chamber $S_{\mathrm{C}}=$ $0.12 \mathrm{~m}^{2}$, and $1 \mathrm{~Pa} \cdot \mathrm{m}^{3} \cdot \mathrm{s}^{-1} \approx 2.42 \times 10^{20} \mathrm{~s}^{-1}$ at the temperature of $300 \mathrm{~K}$ are used. In the FESTA experiments, the out flux released from the test chamber is about $3.6 \times 10^{10} \mathrm{~s}^{-1}$, which is smaller than the theoretical value of $\Gamma_{\text {outC }}=$ $7.3 \times 10^{11} \mathrm{~s}^{-1}$. Since the hydrogen flux released from the wall of QUEST, $\Gamma_{\text {outQ }}$, is obtained from the peak value shown in Fig. 2, the hydrogen flux released from the sample is predicted as:

$$
\Gamma_{\text {outS }}=\Gamma_{\text {outQ }} \frac{S_{\mathrm{S}}}{S_{\mathrm{Q}}} \approx 4.5 \times 10^{13}\left(\mathrm{~S}^{-1}\right) .
$$

$\Gamma_{\text {outs }}$ is about a hundred times larger than $\Gamma_{\text {outC }}$, so $\Gamma_{\text {outC }}$ is also negligible. As a result, we expect to be able to measure dynamic retention released from the targeted sample using FESTA.

\section{Experimental Measurement Using FESTA}

\subsection{FESTA experiments}

During the 2019 S/S campaign, in the FESTA experiments, plasma discharges were operated at a hot wall temperature of $473 \mathrm{~K}$. The plasma electron temperature was around $60 \mathrm{eV}$ and the electron density was about $3 \times 10^{17} \mathrm{~m}^{-3}$.

When the sample is exposed to the plasma, the gate valves 1 and 2 are open and the test chamber and the QUEST vacuum vessel are connected so that fuel particles including charge exchange hydrogen atoms go back and forth through the gate valve 2 . The charge exchanged fuel particles have inherently high energy, so they are easily absorbed by the test chamber wall. After the isolation of the test chamber, the wall-stored fuel particles are released. We call this plasma-induced background.

To remove the plasma-induced background, a mock operation is performed without sample. The results are shown in Fig. 4. The plasma discharges are started at $t=$ $0 \mathrm{~s}$. The hydrogen pressure in the test chamber is constant before closing the gate valves. After the test chamber was isolated, the hydrogen pressure began to increase. It was found when measuring hydrogen pressure in the isolated chamber that there is clear difference in hydrogen pressure for the cases with and without exposure to a plasma discharge. The green dotted line in Fig. 4 shows the test chamber pressure when the chamber is not exposed to a plasma discharge. The large increase in the test chamber pressure for plasma discharges 39554 and 39555 show that hydrogen particles were greatly occluded during plasma discharge by the wall of the test chamber.

\subsection{Model to remove the plasma-induced background}

To calculate the plasma-induced background, a model was developed as shown in the following equations:

$$
\begin{aligned}
& V_{\mathrm{C}} \frac{\mathrm{d} y(t)}{\mathrm{d} t}=0.03 \times 10^{-6} H_{\mathrm{W}}(t)^{2}+0.87 \operatorname{gam}(t) \\
& \quad+\text { outgas }+\operatorname{pump}\left(V_{v}(t)-y(t)\right) \\
& \quad-\text { wallpump }(t) y(t)\left(1-\frac{S_{\mathrm{W}}(t)}{S_{\mathrm{W} 0}}\right) \\
& \frac{\mathrm{d} H_{\mathrm{W}}(t)}{\mathrm{d} t}=0.13 \operatorname{gam}(t)-0.03 \times 10^{-6} H_{\mathrm{W}}(t)^{2}
\end{aligned}
$$



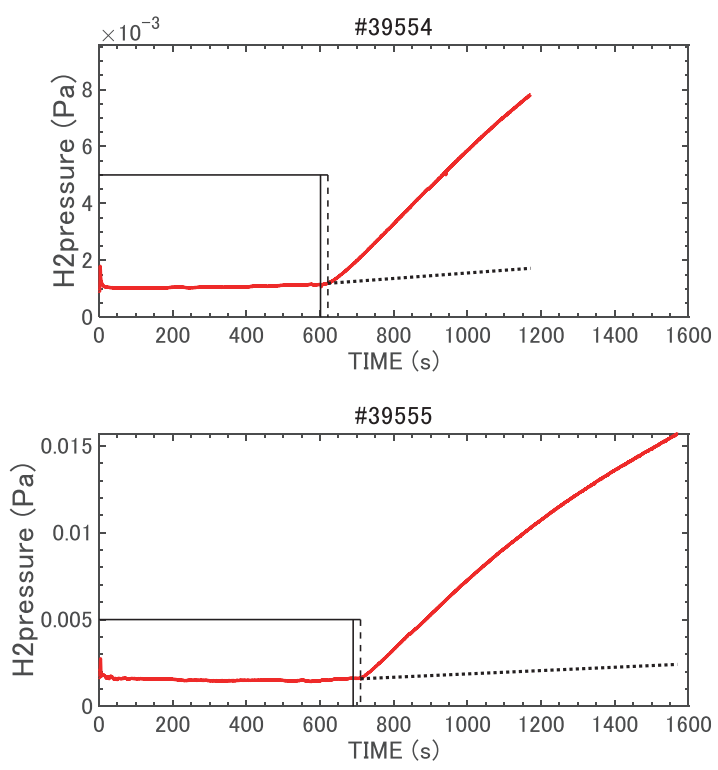

Fig. 4 Time evolution of hydrogen pressure measured with mock operation of FESTA in \#39554 (top) and \#39555 (bottom). The duration of plasma discharges is shown by the black solid square line and the time of closing gate valve 1 and 2 is indicated by the black broken line. The out gas released from the wall of the isolated chamber without a plasma discharge is shown by the black dotted line. The red line shows the pronounced outgassing of the test chamber walls after it is exposed to a plasma discharge.

$$
\frac{\mathrm{d} S_{\mathrm{W}}(t)}{\mathrm{d} t}=\text { wallpump }(t) y(t)\left(1-\frac{S_{\mathrm{W}}(t)}{S_{\mathrm{W} 0}}\right) .
$$

Here $y(t)$ is hydrogen molecules per volume in the test chamber, $H w(t)$ is hydrogen atoms stored in the test chamber, $S w(t)$ is hydrogen atoms absorbed on the test chamber walls and $V v(t)$ is hydrogen molecules per volume in the QUEST vacuum vessel. $\operatorname{gam}(t)$ is the charge exchange neutral flux from QUEST plasma which is proportional to $\mathrm{H} \alpha$ intensity $\mathrm{I}_{\mathrm{H} \alpha}$ that is controlled and kept at a constant value with a developed feedback system $[17,18]$ so that during plasma discharge $\operatorname{gam}(t)$ is constant otherwise it is zero. outgas refers to the outgassing which is always released from the test chamber wall and it is a constant during operation of FESTA when it is connected to the QUEST vessel. pump refers to the conductance between QUEST vacuum vessel and the test chamber and it is zero after gate valve 2 is closed. wallpump $(t)$ is the surface adsorption coefficient of the wall of test chamber, and it is constant when the test chamber is not exposed to plasma, and zero during plasma exposure. The coefficients in these equations are obtained by data fitting to the results obtained from the mock operation as shown in Fig. 5 .

It should be noted that the model does not take diffusion into the test chamber material into consideration. This is because the duration of the FESTA measurement is significantly shorter than time constant of diffusion dominant phenomena. Therefore, we assume the surface density of

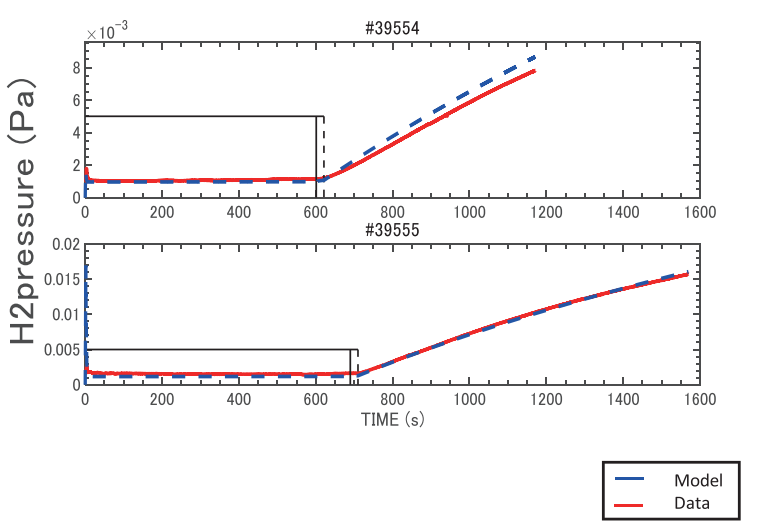

Fig. 5 Hydrogen pressure data without sample and model from the start of plasma discharge to the end of measurement in \#39554 (top) and \#39555 (bottom).

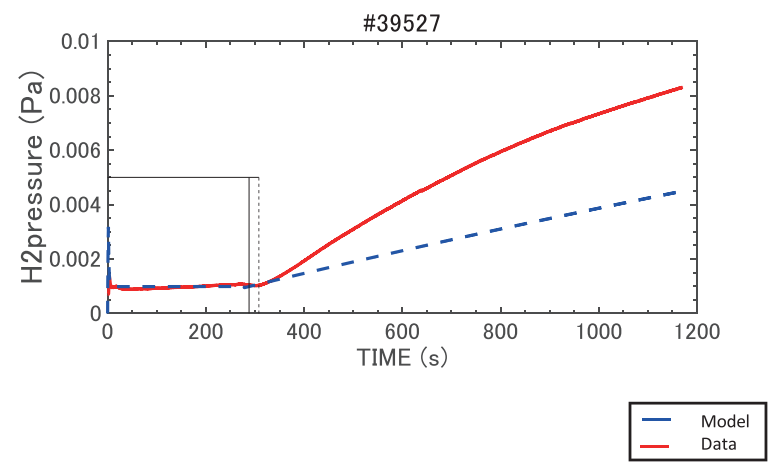

Fig. 6 Hydrogen pressure data with sample and model from the start of plasma discharge to the end of measurement in \#39527. The timing of closing the gate valve 1 and 2 is indicated by the dotted line. The duration of plasma discharge is shown by the solid line.

fuel particles is proportional to the number of wall-stored fuel particles $H_{\mathrm{w}}(t)$ and the surface recombination is expressed by $H_{\mathrm{w}}(t)^{2}$.

The background model predicts a significant out flux from the plasma-exposed sample as shown in Fig. 6. The sample is chemical polished and the duration of plasmaexposure is $288 \mathrm{~s}$. This indicates that dynamic retention released from the QUEST plasma-exposed sample can be measured by using such a background model.

\section{Discussion}

As described above, FESTA has the capability to directly measure dynamic retention of prepared samples. In the experiment reported in the paper, the sample made from stainless steel type $316 \mathrm{~L}$ was used. It is well-known that dynamic retention released from samples with redeposition layer is expected to be completely different from samples without the redeposited layer [9]. Most of PFWs on QUEST was covered with thin redeposited layer induced by plasma wall interaction $[9,10]$. So, one should 
be able to use the plasma-exposed sample to understand the fuel particle balance in real fusion experimental devices. The hydrogen barrier model for metal PFWs has been proposed by K. Hanada [1] and the model should be examined using FESTA. The procedure for the investigation is as follows.

The out flux $\gamma_{\text {out }}$ released from plasma-exposed sample per unit area due to surface recombination is the product of the recombination coefficient $\mathrm{k}$ and the square of hydrogen atom density $C$ given as follows:

$$
\Gamma_{\text {out }}=k C^{2} \text {. }
$$

In the hydrogen barrier model [1], in which there is a barrier between the redeposited layer generated by PWI and the metal material, the hydrogen out flux from the sample is $\Gamma_{\text {out }}=\gamma_{\text {out }} S=\frac{k}{S d^{2}} H_{\text {wall }}^{2}$, so Eq. (1) could be solved as shown,

$$
H_{\mathrm{wall}}=\sqrt{\frac{\gamma_{\mathrm{in}}}{\frac{k}{S d^{2}}}} \tanh \left(\sqrt{\Gamma_{\mathrm{in}} \frac{k}{S d^{2}}} t\right) .
$$

Using FESTA, it can be confirmed whether the number of hydrogen particles released matches Eq. (12) by changing the sample area, material, and the thickness of redeposited layer. So, it should be possible to examine the validity of the hydrogen barrier model. In addition, the recombination coefficient $\mathrm{k}$ can also be experimentally determined, which can contribute to the construction of the dynamic retention model.

\section{Summary}

The FESTA system was developed and successfully tested on QUEST plasma discharges. The LabVIEW control interface worked well, and the system can now support experiments on QUEST. During the experiments the neutral hydrogen pressure contribution from the plasmainduced background was observed and was found to be much more complex than expected. To deduct this background neutral pressure contribution, a model based on out flux of particles which are caused by the fuel particles oc- cluded by the wall of test chamber during plasma exposure was developed using a mock operation of FESTA during which the empty test chamber was exposed to the QUEST plasma, and it was found that the model can well-estimate the plasma-induced background contribution. Finally, we were able to obtain the dynamic retention of the targeted sample exposed by the QUEST plasma using FESTA.

\section{Acknowledgement}

This work was supported by QUEST team. This work was also supported by a Gran-in-Aid for JSPS Fellows (KAKENHI Grant Number 16H02441, 19H05526) and the NIFS Collaboration Research Program (NIFS05KUTRO14, NIFS19KUTR136). This work was also supported in part by a Grant-in-Aid for JSPS Fellows (KAKENHI Grant Number 19H05526), the Collaborative Research Program of the Research Institute for Applied Mechanics, Kyushu University and the JSPS-NRF-NSFC A3 Foresight Pro-gram in the field of Plasma Physics (NSFC: No. 11261140328). I am also grateful to Dr. Roger Raman from University of Washington, Seattle, for discussing and giving me lots of meaningful advice about the manuscript.

[1] K. Hanada et al., Nucl. Fusion 57, 126061 (2017).

[2] D. van Houtte et al., Nucl. Fusion 44, L11 (2004).

[3] S. Brezinsek et al., Nucl. Fusion 53, 083023 (2013).

[4] S. Brezinsek, J. Nucl. Mater. 463, 11 (2015).

[5] T. Loarer et al., Nucl. Fusion 47, 1112 (2007).

[6] V. Philipps et al., J. Nucl. Mater. 438, S1067 (2013).

[7] K. Hanada et al., Plasma Fusion Res. 5, S1007 (2010).

[8] K. Hanada et al., Plasma Sci. Technol. 18, 1069 (2016).

[9] K. Hanada et al., Nucl. Fusion 59, 076007 (2019).

[10] Z. Wang et al., Rev. Sci. Instrum. 88, 093502 (2017).

[11] Y. Oya et al., Fusion Eng. Des. 146, 1480 (2019).

[12] N. Ohno et al., J. Plasma Fusion Res. 90, 59 (2014).

[13] T.J. Finlay et al., J. Nucl. Mater. 443, 145 (2013).

[14] Y. Nakamura et al., J. Nucl. Mater. 438, S1036 (2013).

[15] I. Takagi et al., J. Nucl. Mater. 386-388, 682 (2009).

[16] S.S. Inayoshi et al., Vacuum 53, 281 (1999).

[17] S. Sharma et al., J. Nucl. Mater. 420, 83 (2017).

[18] M. Hasegawa et al., Fusion Eng. Des. 112, 699 (2016). 